\title{
Atherosclerosis risk factors among cardiovascular in hospital patients treated in Montenegro
}

\author{
Goran Nikolic $^{1}$, Agima Ljaljevic ${ }^{2}$, Ljilja Music ${ }^{1}$, Aneta Boskovic ${ }^{1}$, Tanja Cadjenovic ${ }^{1}$ \\ ${ }^{1}$ Klinički centar Crne Gore Ljubljansa bb, 20000 Podgorica, Montenegro \\ ${ }^{2}$ Institute for Public Health of Montenegro
}

Corresponding author: Goran Nikolic: Ljubljansa bb, 20000 Podgorica Montenegro E-mail: goran.nikolic@kccg.me

Project "The degree of development of atherosclerosis in cardiovascular patients treated in Montenegro" was done under the sponsorship of Montenegrin Academy of Arts and Sciences 2010-2012 year.

\begin{abstract}
Objectives: Consequence of atherosclerosis is very often disease in Montenegro. Our goal is determining the frequency of certain risk factors of atherosclerosis in the patients treated of cardiovascular disease in Montenegro

Materials and patients: Between January2011 and December 2012, 1280 patients enrolled in study. The reason of hospitalization were treating of consequence of atherosclerosis, presented as cardiovascular disease. Such patients findings were noted: age, sex, BMI (obesity) cholesterol level (with fractions), blood pressure, presence of diabetes, smoking. In all patients Framing risk score of cardiovascular events was calculated. Comparison with literature data were performed.

Conclusion: The most dominant risk factors that increase the development of atherosclerosis in Montenegro are cigarette smoking, dyslipidemia, and obesity. In Montenegro, the clinical manifestations of atherosclerosis occur at least 10 years earlier than in most developed countries
\end{abstract}

Key words: Smoking, Risk Factors, Atherosclerosis, Cardiovascular Disease, Risk Factors

\section{Introduction}

Definition: Atherosclerosis is a progressive, chronic inflammatory disease of the blood vessels (arteries) in which the walls of the blood vessels become thickened and hardened by "plaques." The plaques are composed of cholesterol and other lipids, inflammatory cells, and calcium deposits.

Atherosclerosis is a diffuse chronic disease of the arteries. Primarily occurs in the intima of the abdominal aorta, its major branches, the lower limb arteries, coronary and cerebral arteries. Atherosclerosis is progressive disease, starts with extracellular and intracellular accumulation of lipids, monocyte / macrophage infiltration, continued with intracellular formation of foam cells, smooth muscle cell proliferation and accumulation of connective-tissue proteins. Clinical atherosclerosis may presents, when the changes of artery arteries progress, as cardiovascular disease. Cardiovascular disease is coronary heart disease (angina pectories, myocardial infarction, sudden cardiac death), cerebrovascular disease (transient ischemic stroke-TIA, stroke), peripheral vascular disease (intermittent claudication, gangrene). These are the most common clinical manifestations. Atherosclerosis can also be presented as renal vascular, carotid disease, mesenteric angina and others.
Atherosclerosis is a complex disease under influence of several risk factors. All risk factors of atherosclerosis are divided into classes: unchangeable and variable. Variable atherosclerosis risk factors include dyslipidemia, hypertension, cigarette smoking, obesity and unchangeable inculude genetic factors, age, and diabetes (1).

The goals of the project:

1. To determine the degree of atherosclerosis in patients treated for cardiovascular disease in Montenegro.

2. To determine the frequency of certain risk factors of atherosclerosis in the patients treated of cardiovascular disease in Montenegro

3. Determine the most frequent risk factors for the development of atherosclerosis among cardiovascular in hospital patients treated in Montenegro.

\section{Materials and patients}

During 2011 and 2012, 1280 consecutive hospitalized patients were involved in study. The patients were hospitalized in Clinical center of Montenegro with aim of treated clinical manifestation of atherosclerosis. In every patient such findings were recorded: age, sex, BMI (obesity) cholesterol level (with 
fractions), blood pressure, presence of diabetes, smoking. In all patients Framing risk score of cardiovascular events was calculated.

\section{Results}

Age distribution is presented on Table 1.

\begin{tabular}{|l|c|c|}
\hline Years & $\begin{array}{c}\text { Number of } \\
\text { patients }\end{array}$ & $\%$ \\
\hline$<40$ & 24 & 1,87 \\
\hline $\mathbf{4 1 - 5 0}$ & 85 & 6,64 \\
\hline $\mathbf{5 1 - 6 0}$ & 436 & 34,06 \\
\hline $\mathbf{6 1 - 7 0}$ & 512 & 40 \\
\hline$>\mathbf{7 0}$ & 223 & 17,42 \\
\hline Sum & 1280 & 99,99 \\
\hline
\end{tabular}

Table 1. Age distribution of patients

In our series there were 805 (62.89\%) men, and 475 (37.1\%) women.

Cholesterol findings is presented on Table 2.

\begin{tabular}{|l|c|c|c|}
\hline \multirow{2}{*}{} & \multicolumn{3}{|c|}{ Cholesterol } \\
\cline { 2 - 4 } & Tot & HDL & LDL \\
\hline mean & 5,3 & 1,4 & 4 \\
\hline min & 3,2 & 0,7 & 6,7 \\
\hline max & 15,5 & 2,6 & 2 \\
\hline SD & 2,1 & 0,41 & 1,18 \\
\hline Sum & 1280 & 1280 & 1280 \\
\hline
\end{tabular}

Table 2. Patients level of Cholestrol

In the our series the average arterial tension was measured to $133 / 83 \mathrm{mmHg}$. ( $\max 240 / 110, \min 100 / 60$ SD $20,94 / 13.68$ )

There were 563 (44.14\%) smokers and 717 (55.855) non smokers among patients

In our series, the overall BMI was 26.6 In our series, the overall BMI was $26.6 \mathrm{~kg} / \mathrm{m} 2$. ( $\min 20.85 \max 30.0$ SD 3.74). Normal BMI is $25.0 \mathrm{~kg} / \mathrm{m} 2$.

In our studied patients diabetes type II was found in 354 (27.65\%) of patients without diabetes was 926 (72.34\%)

Mean Framingam risk score was measure to $15 \%$ (min 2 max 53).That mean moderate risk of cardiovascular events in 10 years

\section{Discussion}

Our study confirmed previous views that clinically manifest atherosclerosis is more common in men than in women (2). The difference is evident in gender less than 55 years. Women, under 55 are probably protecte by estrogen against the development of atherosclerosis. After this age, the incidence is equal in persons of different sexes.

Dyslipidemia is one of the main factors in development atherosclerosis. This occurs in increased entry of fat cholesterol. The cells haven't the ability of cholesterol synthesis (an important component of cell membranes, myelin coating and steroid hormones), but it is synthesized in the liver. From the liver to the cells, cholesterol is transported by lipoproteins (consists of protein, cholesterol, triglycerides and phospholipids) in the form of LDL (low density lipoproteins). Synthesis of cholesterol in liver depends of intake, but cells doesn't accept and accumulate whole production of cholesterol. The cell is released cholesterol in the form of HDL (high density lipoprotein), which accumulates in the liver. Total cholesterol is the sum of plasma LDL and HDL cholesterol. Their relationship is a major factor in the impact assessment of cholesterol in atherosclerosis than the total value of the plasma cholesterol. High LDL cholesterol (> $3.4 \mathrm{mmol} / \mathrm{L}$ ) occurs in increased food intake, and keeps increased risk of atherosclerosis (3). Low HDL means reduced cell release of cholesterol and also increased risk $(<1.8 \mathrm{mmol} / \mathrm{L})$ of atherosclerosis. Desired level of total cholesterol in our study was below $5.2 \mathrm{mmol} / \mathrm{L}$ Total cholesterol is $5.3 \mathrm{mmol} / \mathrm{L}$. It is just above the accepted value but most of these patients were on statins anti cholesterol therapy and registered cholesterol level includes effects of therapy.

Hypertension and atherosclerosis in mechanisms of development bouth of them are linked. Atherosclerosis leads arteries to become stiff and firm, which in further course leads to hypertension, which continue to damages the endothelium of arteries and hypertension increase. This is the classical cascade formation in progression of hypertension. In the our series the average tension was measured to $133 / 83 \mathrm{mmHg}$. That means pre-hypertension state. We shouldn't forget that in the series $53.36 \%$ of patients were on anti hypertensive therapy.

Smoking is a variable risk factor.of atherosclerosis. Cigarette smoking contributes significantly to the development of atherosclerosis. Cigarettes contain many prooxidants that can directly affect the oxidative stress of endothelial cells. Oxidative stress is one of the predictors and accelerators of atherosclerosis. Smoking is a social-medical, medical and preventive treatment problem. Control of smoking need extensive work of social and state resources.

Among our patients who were hospitalized with clinically manifest atherosclerosis were $44.14 \%$ smokers Table 4 According to available data among the population of Great Britain in 2010, there were $21 \%$ smokers. and in the USA in 2009 , it was noted $20 \%$ of smokers in the population (4).

This difference was statistically highly significant. It should be noted that in our series percentage of cardiovascular patients smokers were higher than percentage of smokers in population in other countries. 
This conclusion should be the alarm for the fight against smoking in our country. It is not rational to treat atherosclerosis consequences if smoking doesn't be excluded as factors which cause atherosclerosis and accelerate it.

Obesity is a variable risk factor for atherosclerosis. BMI (body mass index) greater than $30 \mathrm{~kg} \mathrm{~m} 2$ a increases the risk of atherosclerosis four times higher in compare with a normal BMI. Currently in Europe 20\% population is obese. According to the trends of incising number of obese people is predicted that in the 21st century obesity become a major risk factor in the development of atherosclerosis. Obesity leads to endothelial dysfunction in the disorder of metabolism of nitric oxide (NO) (5). In our series, the overall BMI was $26.6 \mathrm{~kg} / \mathrm{m} 2$. Above normal $(25 \mathrm{~kg} / \mathrm{m2})$ was ascertained 547 patients, which makes $42.73 \%$ of all. It can be conclude that almost half of patients with clinically manifest atherosclerosis had increased body mass.

Numerous studies indicate that there is accelerated atherosclerosis in diabetic patients. In diabetes there is endothelial dysfunction, which is the basis of atherosclerosis (6). Diabetes increases the risk of atherosclerosis 2-4 times. Early detection and adequate treatment of diabetes type II is prevention of cardiovascular events in these patients.

Framingam risk score represents the formula of calculating the risk of cardiovascular events (myocardial infarction and stroke) in the period of 10 years. (7) Framingam score is rated up to $10 \%$ as low risk, medium risk $10-20 \%$ and over $20 \%$ is high risk of cardiovascular events. According to the data from our patient series Framingam middle score is $15 \%$. According to the data our patients were among those with a high risk of developing cardiovascular event in 10 years. In the same series 374 patients were registered as high risk (over 20\%).

\section{Conclusion}

Atherosclerosis in our patients are clinically manifested to 10 years earlier than in other areas.

1. Atherosclerosis is very important a cause of disease is in Montenegro.

2. All of the examined risk factors for atherosclerosis are increased in patients with clinically manifest signs of atherosclerosis in Montenegro. Obesity, cholesterol levels, blood pressure, smoking, risk factors are present

3. Of the risk factors that increase the development of atherosclerosis in Montenegro are the most dominant are cigarette smoking, dyslipidemia, and obesity.

4. In the assessment of cardiovascular risk (Framingam risk score) there is a high risk of cardiovascular events (myocardial infarction, stroke, gangrene)

Unplanned achieved goal of research is very important. In Montenegro, the clinical manifestations of atherosclerosis occur at least 10 years earlier than in most developed countries

This research was conducted with the primary aim of encouraging organized national campaign in prevention of atherosclerosis. The best way in organized prevention of atherosclerosis is to establish national body for combat against atherosclerosis widely supported by health authorities and relevant ministries, civil society and the media. The research proved that the patients with cardiovascular disease are 10 years younger than in developed countries. The most common risk factor for atherosclerosis in Montenegro, are dyslipidemia, obesity and smoking.

\section{References}

1. Markus J, Jorma V, Mika K, Leena T, Tomi L, Nina H. Lifetime risk factors and progression of carotid atherosclerosis in young adults: the Cardiovascular Risk in Young Finns study. Eur Heart J 2010;31:1745-1751. doi: 10.1093/eurheartj/ehq141

2. Bairey MN, Shaw L, Reis SE, Bittner V, Kelsey SF, Olson M, et all . Part II: Gender Differences in Presentation, Diagnosis, and Outcome With Regard to Gender-Based Pathophysiology of Atherosclerosis and Macrovascular and Microvascular Coronary Disease. J Am Coll Cardiol 2006;47 doi: 10.1016/j.jacc.2004.12.084

3. Khera AV, Cuchel M, De LLM, Rodrigues A, Burke MF, Jafri K, Rader DJ. Cholesterol efflux capacity, high-density lipoprotein function, and atherosclerosis. N Engl J Med 2011;364(2):12735. PubMed PMID: 21226578. doi: 10.1056/NEJMoa1001689

4. Available from:

http://www.census.gov/compendia/statab/cats/health_nutrit ion.html

5. Williams IL, Wheatcroft SB, Shah AM, Kearney MT. Obesity, atherosclerosis and Vascular endothelium, mechanisms of reduced nitric oxide bioavailability in obese humans. Int J Obes Relat Metab Disord 2002;26(6):754-764.

6. Hegazi RAF, Sutton-Tyrrell K, Evans RW, Lewis KH, Belle S, Yamamoto M, Kelley DE. Relationship of Adiposity to Subclinical Atherosclerosis in Obese Patients with Type 2 Diabetes. Obesity Research 2003;11:1597-1605. doi: 10.1038/oby.2003.212

7. Wilson PW, D.R.B. , Levy D, Belanger AM, Silbershatz H, Kannel WB. Prediction of coronary heart disease using risk factor categories. Circulation 1998;97(18):1837-47. PubMed PMID: 9603539. doi: 10.1161/01.CIR.97.18.1837 